\title{
RESEARCH HIGHLIGHT Mitochondrial DNA synthesis fuels NLRP3 inflammasome
}

\author{
Rebecca C. Coll $\mathbb{D}^{1}$, Caroline L. Holley $\mathbb{D}^{1}$ and Kate Schroder $\mathbb{D}^{1}$ \\ Cell Research (2018) 28:1046-1047; https://doi.org/10.1038/s41422-018-0093-8
}

\begin{abstract}
Mitochondria-derived signals are associated with NLRP3 inflammasome activation. A recent study in Nature by Zhong et al. shows that inflammasome priming drives new mitochondrial DNA synthesis, and proposes this to be a prerequisite for NLRP3 inflammasome signaling.
\end{abstract}

NLRP3 is a cytosolic pattern recognition receptor that is activated in response to a broad range of pathogen-derived and endogenous agents. NLRP3 assembles an inflammasome complex with ASC to activate caspase- 1 , which drives the maturation and secretion of the pro-inflammatory cytokines IL-1 $\beta$ and IL-18, and concurrently induces Gasdermin-D-dependent cell lysis. NLRP3 contributes to many acquired and hereditary inflammatory diseases (e.g., Alzheimer's, gout, Muckle-Wells syndrome). ${ }^{1}$ The mechanism of NLRP3 activation is complex, and tightly regulated by processes broadly categorized into two stages. Signal one, the "priming" or "licensing" signal, serves two functions: it enhances expression of NLRP3 and pro-IL-1 $\beta$, and triggers NLRP3 posttranslational modifications. Signal two then triggers NLRP3 activation via pathways involving potassium efflux and/or mitochondria, ${ }^{1}$ resulting in inflammasome assembly and signaling.

Mitochondria are closely entwined with the macrophage inflammatory response, and a number of previous studies have linked NLRP3 to mitochondria. Mitochondrial disruption caused by NLRP3 stimuli leads to generation of reactive oxygen species (ROS) and exposure of mitochondrial DNA (mtDNA) to the cytoplasm., ${ }^{2,3}$ Cytoplasmic mtDNA co-localizes with NLRP3 and enhances IL-1 $\beta$ secretion, while oxidized mtDNA is a more potent inducer of IL-1 $\beta$ production. ${ }^{4}$ Until now, mtDNA has only been linked to the activation signal in NLRP3 inflammasome responses. In a recent study published in Nature, Zhong et al. ${ }^{5}$ demonstrate that Toll-like receptor (TLR)-dependent priming signals initiate the synthesis of new mtDNA, and propose that this is required for NLRP3 activation.

Consistent with previous reports, Zhong et al. observed that NLRP3 activators triggered mtROS production and loss of mitochondrial membrane potential, regardless of cellular priming status. When cells were primed with the TLR4 ligand lipopolysaccharide (LPS), NLRP3 activators also triggered an increase in oxidized mtDNA release into the cytosol. LPS stimulation was associated with a 2-3-fold increase in mtDNA copy number but not mitochondrial mass as measured by mitochondrial protein levels. LPS triggered mtDNA synthesis via the TLR4 adapters MyD88 and TRIF, with an early requirement for MyD88 and a dominant role for TRIF at later time points.

The authors used genetic approaches to reduce mtDNA levels by deleting TFAM (transcription factor A, mitochondrial) or knocking down DNA polymerase- $\gamma$ (POL $\gamma)$ in myeloid cells. Macrophages deficient in TFAM or POLY showed reduced NLRP3 activation but normal responses to AIM2 inflammasome stimulation, suggesting a specific requirement for mtDNA in the NLRP3 pathway.

The authors further examined the mechanism linking TLR4 stimulation to increased mtDNA replication. LPS signaling activates the transcription factor, interferon regulatory factor 1 (IRF1), and the authors report that IRF1 was required for LPSstimulated mtDNA upregulation. Irf1 deficiency diminished oxidized mtDNA and suppressed NLRP3 responses. In vivo, Irf1 deficiency suppressed NLRP3-driven inflammatory responses during murine endotoxemia and alum-induced peritonitis.

IRF1 was functionally linked to the mitochondrial deoxyribonucleotide kinase UMP-CMPK2 (CMPK2). IRF1 bound to the promotor of Cmpk2 and was required for LPS-driven CMPK2 upregulation. The resultant increase in CMPK2 catalytic activity is proposed to enhance the supply of dNTP for LPS-stimulated mtDNA synthesis, while dNTP levels are restrained by the nucleoside triphosphate hydrolase SAMDH1. SAMDH1 deficiency enhanced LPS-stimulated mtDNA synthesis and NLRP3-driven IL-1 $\beta$ secretion, whereas Cmpk2 knockdown reduced mtDNA levels and oxidized mtDNA release into the cytosol, and suppressed NLRP3 responses, similar to the earlier experiments with $I r f 1^{-1-}$ macrophages. Importantly, overexpression of wild-type but not catalytically-inactive CMPK2 in Irf1 ${ }^{-1-}$ cells rescued LPS-induced mtDNA synthesis.

Zhong et al. observed that $I r 1^{-1-}$ macrophages were not defective in IL-1 $\beta$ release in response to LPS transfection. It is well established that IL-1 $\beta$ release induced by cytosolic LPS requires both caspase-11 and non-canonical NLRP3 activation. ${ }^{1}$ Therefore, the observation from Zhong and co-workers that IRF1 is essential for IL-1 $\beta$ production induced by canonical NLRP3 signaling but dispensable for caspase-11-driven IL-1 $\beta$ production, is somewhat surprising. Previous studies have placed IRF1 upstream of caspase11 and AIM2 activation, but found that IRF1 was dispensable for NLRP3 activation. ${ }^{6}$ The role of IRF1 in NLRP3 activation thus remains unclear.

Zhong et al. report a new pathway in which TLR signaling stimulates new mtDNA synthesis via IRF-1 and CMPK2, and propose that the resultant increase of mtDNA enhances the release of oxidized mtDNA into the cytosol to activate NLRP3 (Fig. 1). These results link mitochondrial function to the poorly understood priming stage of NLRP3 activation, and expand the known mechanisms of TLR priming of NLRP3 responses. Oxidized cytosolic mtDNA may thus fuel a stronger NLRP3 response to microbial stimuli sensed by TLRs (such as LPS) than to a stimulus such as TNF, which is known to prime a less robust NLRP3 response. ${ }^{7}$ It will be interesting to examine whether endogenous priming stimuli, such as the cytokines TNF and IL-1, differentially regulate mtDNA synthesis and thus differ in their capacity to modulate NLRP3 responses.

Previous studies have questioned whether mitochondrial damage and mtROS are indeed universally required for NLRP3

${ }^{1}$ Institute for Molecular Bioscience and IMB Centre for Inflammation and Disease Research, The University of Queensland, St Lucia, QLD, Australia

Correspondence: Rebecca C. Coll (r.coll@imb.uq.edu.au) or Kate Schroder (k.schroder@imb.uq.edu.au)

Published online: 3 October 2018 


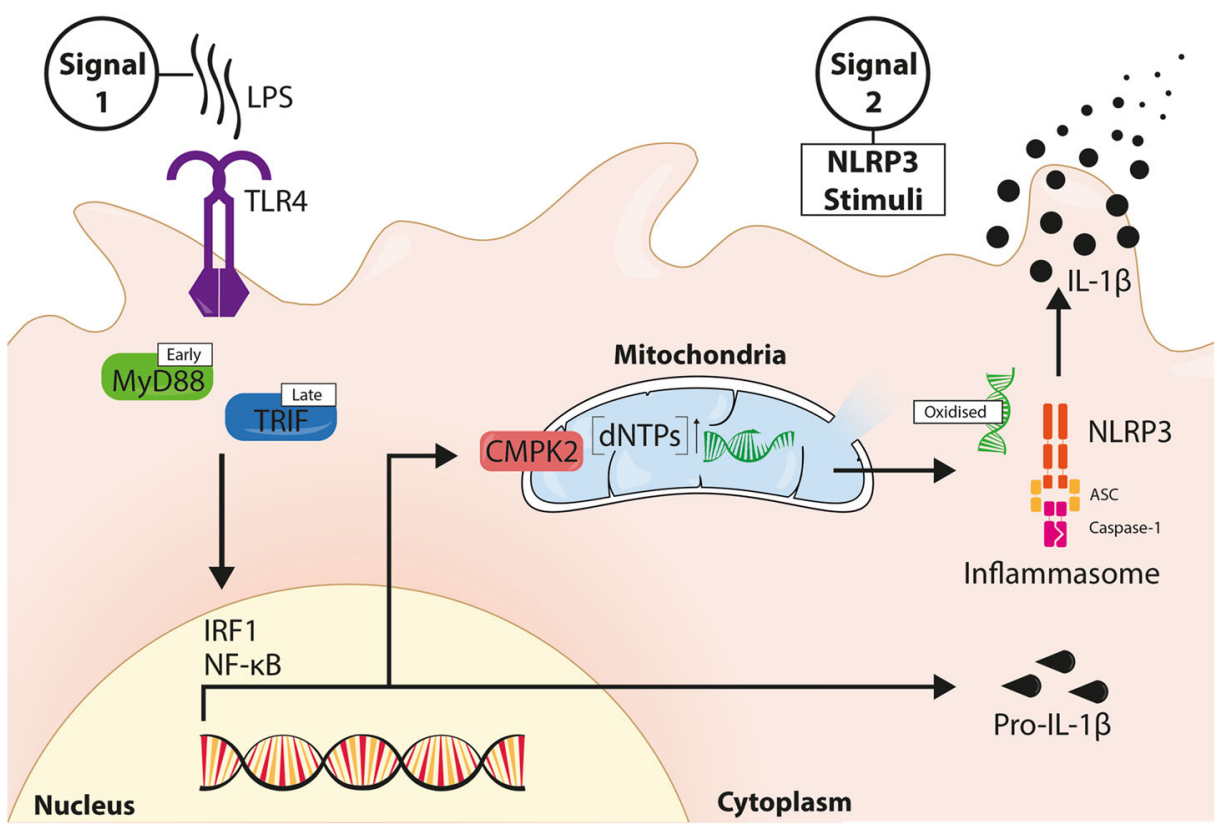

Fig. 1 LPS-induced new mtDNA contributes to NLRP3 signaling. LPS binds TLR4 and induces receptor ligation, driving MyD88- and TRIFdependent activation of transcription factors IRF1 and NF-KB. NF-kB induces the expression of NLRP3 and pro-IL-1 $\beta$ during inflammasome priming. IRF1 drives expression of the nucleoside monophosphate kinase CMPK2, which translocates to mitochondria and enhances the supply of dNTPs for mtDNA synthesis. NLRP3 signal 2 causes mitochondrial dysfunction and ROS production. Oxidized mtDNA directly binds NLRP3 to promote NLRP3 inflammasome assembly and signaling, leading to IL-1 $\beta$ secretion

activation. ${ }^{8,9}$ As previous research using $\mathrm{NIrp3}^{-1-}$ macrophages has placed mitochondrial damage downstream of NLRP3 signaling, $^{10}$ it will be important to establish whether mtDNA release into the cytosol occurs up or downstream of inflammasome signaling, e.g., by using $\mathrm{NIrp}^{-1-}$ cells. Given a previous report of AIM2-dependent mitochondrial damage, ${ }^{11}$ it is surprising that Zhong et al. do not observe mtROS generation or mtDNA release upon AIM2 inflammasome activation. If as the authors suggest, mtDNA is not released into the cytosol during inflammasome-mediated cell death, but is transported to the cytosol prior to NLRP3 inflammasome activation, this raises the intriguing question of how mtDNA is delivered from mitochondria to the cytosol. It also raises the question of how this new pathway for NLRP3 activation integrates with other characterized requirements for NLRP3 activation, such as interaction with NEK7, or cytosolic ionic flux. ${ }^{1}$

Zhong et al. suggest that NLRP3 specifically recognizes oxidized DNA, whereas AIM2 senses non-oxidized DNA. These observations are intriguing, but should be carefully examined using AIM2- and NLRP3-deficient cells to confirm that these sensors operate in a non-redundant manner to recognize cytosolic DNA. It will be interesting to examine whether this mitochondrial priming mechanism also occurs: (1) during rapid priming, where the cell is stimulated with LPS at the same time as, or immediately before, the NLRP3 agonist ${ }^{7,12}$ and (2) in other cell types such as neutrophils, which are major contributors to IL-1 $\beta$ production in vivo and reportedly contain relatively few mitochondria.

In summary, this study ${ }^{5}$ demonstrates that the NLRP3 priming stimulus LPS induces new mtDNA synthesis to enhance oxidized mtDNA release into the cytosol, providing fuel for NLRP3 activation and enhanced inflammatory responses.

\section{REFERENCES}

1. Mangan, M. S. J. et al. Nat. Rev. Drug Discov. 17, 588-606 (2018).

2. Zhou, R. et al. Nature 469, 221-225 (2011).

3. Nakahira, K. et al. Nat. Immunol. 12, 222-230 (2011).

4. Shimada, K. et al. Immunity 36, 401-414 (2012).

5. Zhong, Z. et al. Nature 560, 198-203 (2018)

6. Man, S. M. et al. Nat. Immunol. 16, 467-475 (2015).

7. Bezbradica, J. S. et al. Cell Mol. Immunol. 14, 118-126 (2017).

8. Munoz-Planillo, R. et al. Immunity 38, 1142-1153 (2013).

9. Allam, R. et al. EMBO Rep. 15, 982-990 (2014).

10. Heid, M. E. et al. J. Immunol. 191, 5230-5238 (2013).

11. Yu, J. et al. Proc. Natl Acad. Sci. USA 111, 15514-15519 (2014).

12. Schroder, K. et al. Immunobiology 217, 1325-1329 (2012). 